\title{
Early experiences with robot-assisted prosthetic breast reconstruction
}

\author{
Sung Jae Ahn ${ }^{1}$, Seung Yong Song ${ }^{1}$, Hyung Seok Park ${ }^{2}$, Se Ho Park ${ }^{2}$, Dae Hyun Lew ${ }^{1}$, Tai Suk Roh ${ }^{1}$, \\ Dong Won Lee ${ }^{1}$ \\ Departments of ${ }^{1}$ Plastic and Reconstructive Surgery and ${ }^{2}$ Surgery, Yonsei University College of Medicine, Seoul, Korea
}

\begin{abstract}
Robotic surgery facilitates surgical procedures by employing flexible arms with multiple degrees of freedom and providing high-quality 3-dimensional imaging. Robot-assisted nipplesparing mastectomy with immediate reconstruction is currently performed to avoid breast scars. Four patients with invasive ductal carcinoma underwent robot-assisted nipple-sparing mastectomy and immediate robot-assisted expander insertion. Through a 6-cm incision along the anterior axillary line, sentinel lymph node biopsy and nipple-sparing mastectomy were performed by oncologic surgeons. The pectoralis major muscle was elevated, an acellular dermal matrix (ADM) sling was created with robotic assistance, and an expander was inserted into the subpectoral, sub-ADM pocket. No patients had major complications such as hematoma, seroma, infection, capsular contracture, or nipple-areolar necrosis. The mean operation time for expander insertion was 1 hour and 20 minutes, and it became shorter with more experience. The first patient completed 2-stage prosthetic reconstruction and was highly satisfied with the unnoticeable scar and symmetric reconstruction. We describe several cases of immediate robot-assisted prosthetic breast reconstruction. This procedure is a feasible surgical option for patients who want to conceal surgical scars.
\end{abstract}

Keywords Robotic surgical procedures / Mammaplasty / Breast implants / Tissue expansion devices

\author{
Correspondence: Dong Won Lee \\ Department of Plastic and \\ Reconstructive Surgery, Yonsei \\ University College of Medicine, 50 \\ Yonsei-ro, Seodaemun-gu, Seoul \\ 03722, Korea \\ Tel: +82-2-2228-2215 \\ Fax: +82-2-393-6947 \\ E-mail:xyphoss@yuhs.ac
}

Received: 9 Jan 2018 • Revised: 21 Jun 2018 • Accepted: 10 Jul 2018

pISSN: 2234-6163 • elSSN: 2234-6171 • https://doi.org/10.5999/aps.2018.00052 • Arch Plast Surg 2019;46:79-83

\section{INTRODUCTION}

Nipple-sparing mastectomy with immediate breast reconstruction has become the preferred surgical option for therapeutic and prophylactic mastectomy because it offers higher patient satisfaction and superior aesthetic outcomes without compromising oncologic safety $[1,2]$. Traditionally, skin envelope incision is inevitable, even in nipple-sparing mastectomy. A new endoscopic technique for nipple-sparing mastectomy has been tried, with oncologic outcomes that were not inferior to those of traditional mastectomy [3]. However, the endoscopic approach remains hampered by a limited degree of freedom, an inadequate dissection angle, and interference between instruments at a single axillary access point [4]. A robotic system with flexible arms with several degrees of freedom that provides high-quality 3-dimensional (3D) images has shown great promise for improving the operative technique and postoperative outcomes in various fields, including head and neck surgery, colorectal surgery, and genitourinary surgery [5]. While recent reports have described in detail the use of robotic breast surgery for nipple- 
sparing mastectomy $[6,7]$, the current study focused on reconstructive techniques with an acellular dermal matrix (ADM) sling using a robotic device. We describe several patients with invasive ductal carcinoma who underwent robot-assisted nipple-sparing mastectomy and implant-based immediate breast reconstruction with satisfactory results.

\section{CASE REPORT}

Although an endoscopic transaxillary approach is a familiar surgical route for plastic surgeons, in the preclinical stage, we performed robot-assisted expander insertion in four breasts from two fresh cadavers. This cadaveric study tested the feasibility of mastectomy and reconstruction, including an assessment of significant considerations such as patient arm position, incision length, incision site, the setup and positioning of patient cart instruments (Intuitive Surgical Inc., Sunnyvale, CA, USA), the type of EndoWrist instruments (Intuitive Surgical Inc.), and how to secure sufficient working space (Fig. 1).

Four patients were referred to the breast cancer clinic for invasive ductal carcinoma. Prosthetic reconstruction was recommended due to their non-ptotic and moderate-sized breasts. Since they expressed a strong desire to avoid breast scars, we proposed robot-assisted nipple-sparing mastectomy and immediate reconstruction using expanders. Operations were carried out using the da Vinci Xi Surgical System (Intuitive Surgical Inc.). A 6-cm-long extramammary axillary incision was made in the axillary fossa, parallel to the anterior axillary line; the resulting scar was invisible in the frontal view and hidden by arm abduction in the lateral view (Fig. 2). Sentinel lymph node biopsy and robot-assisted nipple-sparing mastectomy were performed by oncologic surgeons [8]. Breast tissue was removed through

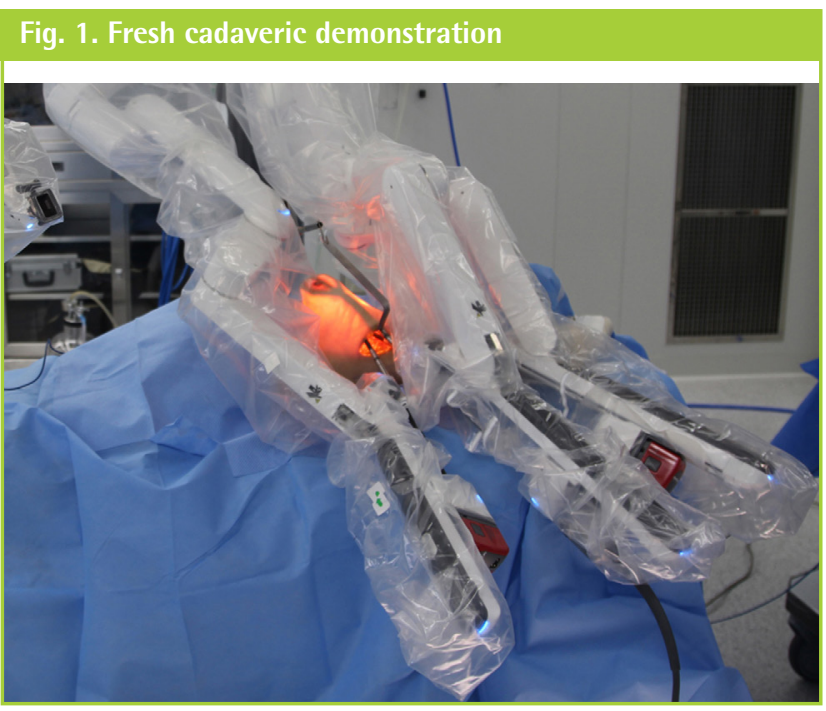

the axillary incision. After mastectomy, the patient cart and EndoWrist instruments were sterilized and redraped for reuse during the reconstruction procedure. The surgeons determined the range of dissection and selected an appropriate expander size considering the specimen weight and chest wall width. Next, the pectoralis major muscle was elevated from the chest wall, beginning from the superolateral border and extending to the nipple level under direct vision. From the nipple level, chest wall curvature interfered with further dissection to the inferomedial border of the muscle. However, the robotic system could provide clear vision and accurate manipulation, and further dissection to the inferomedial origin of the muscle was therefore performed using the robotic system. The center column and camera arm of the patient cart axis was aligned from the axilla to the inferomedial sternal origin of the muscle. The working space was secured with a long and wide blade of an external retractor (modified Chung retractor) that was customized for minimizing skin flap tension [9]. An 8-mm Maryland Bipolar Forceps EndoWrist instrument (Intuitive Surgical Inc.) was fitted on the left robotic arm for traction and counter-traction, and an 8-mm Monopolar Permanent Cautery Spatula EndoWrist instrument (Intuitive Surgical Inc.) was used on the right robotic arm for dissection (Fig. 3A). The inferior origin of the pectoralis major was then cut, and an ADM sling was made with Vicryl interrupted sutures. The deep sutures were made with a Mega SutureCut Needle Driver (Intuitive Surgical Inc.) on the right robotic arm (Fig. 3B). After preparing a subpectoral pocket for the mammary expander, a 200-mL negative drainage system was introduced, and copious irrigation with saline containing antibiotics was performed. An expander was inserted into the subpecto-

\section{Fig. 2. Axillary incision}

A 6-cm incision was made on the axilla. Nipple-sparing mastectomy and prosthetic reconstruction were performed by oncologic surgeons and plastic surgeons, respectively.

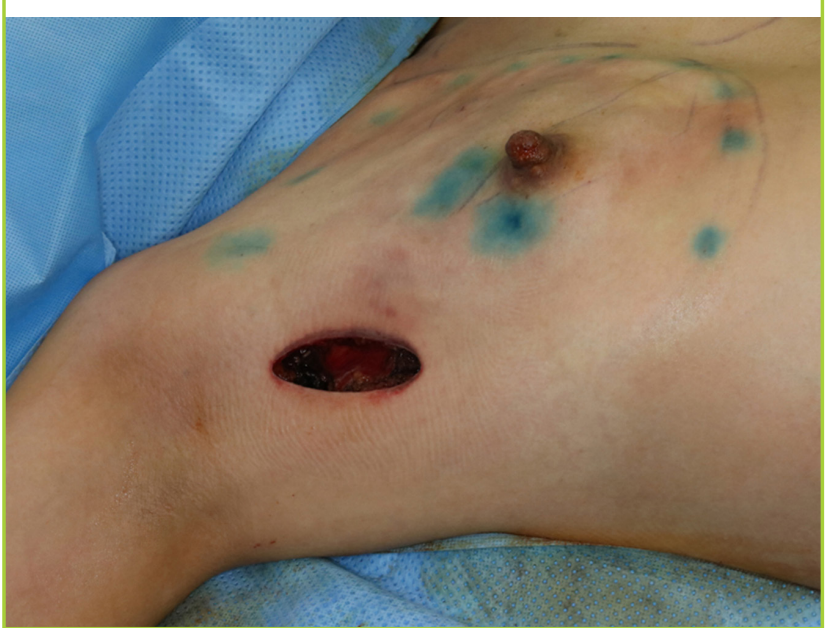




\section{Fig. 3. Robotic reconstruction}

(A) Subpectoral plane dissection. (B) Acellular dermal matrix sling fixation.
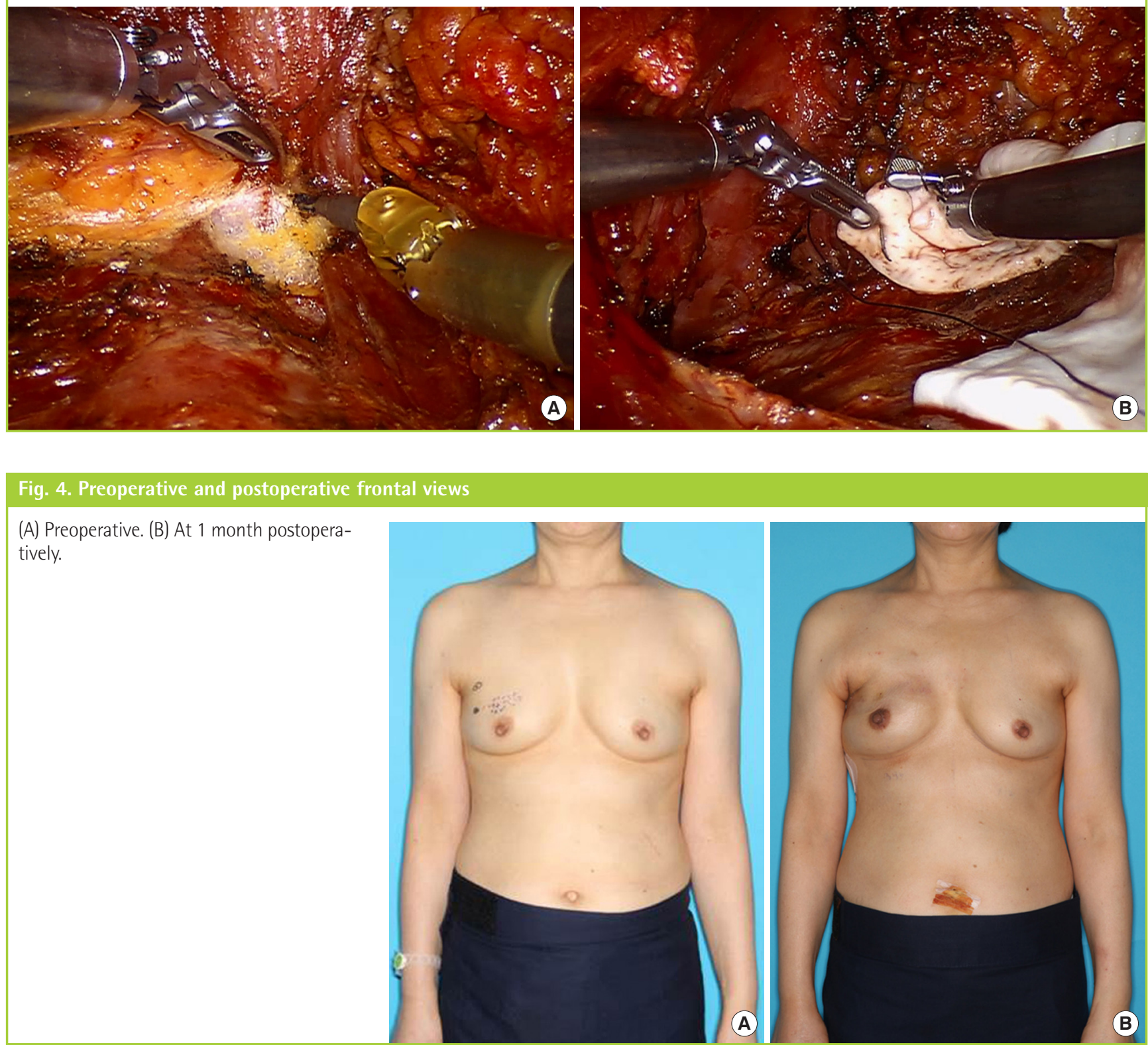

ral pocket. Intraoperatively, the expander was inflated as appropriate, considering skin tension.

\section{Assessment}

A digital camera was used to take preoperative and postoperative clinical photographs. Patient satisfaction was assessed with the breast reconstruction module of the BREAST-Q. A control group was selected from age- and cancer stage-matched patients who underwent nipple-sparing mastectomy with a conventional method and reconstruction with 2-staged prosthetic reconstruction without a contralateral balancing procedure.

\section{Results}

No patients had major complications such as hematoma, seroma, infection, capsular contracture, or nipple-areolar necrosis. One patient experienced mastectomy flap congestion, which subsided without a major operation. The mean operation time for expander insertion was 1 hour and 26 minutes. The first case took 1 hour and 47 minutes, while the subsequent three cases took approximately 1 hour and 20 minutes, indicating that the operation time decreased with the learning curve.

Only the first patient completed first- and second-stage prosthetic reconstruction, while other patients were preparing for second-stage reconstruction at the time of publication; the first 


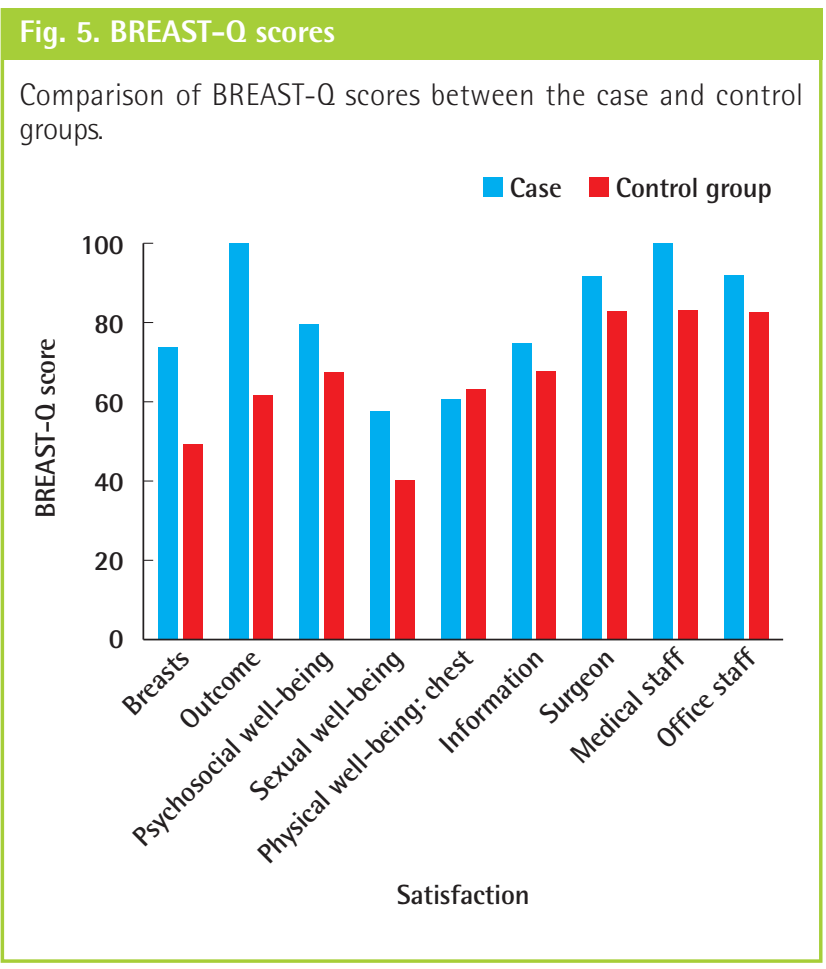

patient was satisfied with her results, especially the unnoticeable scar. Preoperative and postoperative clinical photos of the patients are shown in Fig. 4. The BREAST-Q scores of the first patient calculated 1 month after second-stage reconstruction were compared with the those of the control group calculated at an average of 35.5 days after second-stage reconstruction (Fig. 5). The score for satisfaction with one's breasts was 73 in the robotic case, compared to a mean of 49.5 in the conventional approach group. Satisfaction with the outcome was also higher in the robotic case ( $100 \mathrm{vs.} \mathrm{a} \mathrm{mean} \mathrm{of} 61$ in the control group). The scores for psychosocial well-being and sexual well-being were 57 and 60 , respectively, in the robotic case, in contrast to mean values of 39.7 and 62.3 in the control group, respectively. However, physical well-being (chest) was similar in the two groups, with a score of 60 in the robotic case and a mean score of 62.5 in the control group. Otherwise, the scores for satisfaction with information, the surgeon, the medical staff, and the office staff were higher in the robotic case $(74,91,100$, and 91 , respectively) than in the control group $(67,82,82$, and 82 , respectively).

\section{DISCUSSION}

Avoiding direct scars on the breast envelope is an important strength of this robotic approach, as doing so contributes to patient satisfaction and improves aesthetic outcomes. This robotic approach is similar to an endoscopic approach, which plastic surgeons commonly use for augmentation mammoplasty.
There are several advantages of the robotic approach, such as clear 3D vision with magnification, 7 degrees of freedom of motion (including in/out, rotation, pitch at wrist, yaw at wrist, pitch at fulcrum, yaw at fulcrum, and grip strength), delicate manipulation, and being easy to learn compared with an endoscopic approach.

Recreating a symmetric inframammary fold is an essential step in breast reconstruction. For this, accurately suturing ADM in an appropriate position in the chest wall is important. When the endoscopic approach is used, it is not easy to suture ADM on the chest wall. To the best of our knowledge, suturing ADM in place has not been discussed in previous reports describing endoscopic or robotic mastectomy and reconstruction. However, with the advantages of the robotic approach, symmetrically suturing ADM is facilitated by the presence of 7 degrees of freedom. These advantages make the robotic device more ergonomic than an endoscopic approach, and it is therefore expected to reduce postoperative complications and to enable better aesthetic outcomes [10]. Robot-assisted free flap reconstruction, including robot-assisted microanastomosis in head and neck reconstruction procedures, has been reported [11]. Similarly, we believe that free flap reconstruction could be possible in breast reconstruction with the advantages of a robotic system.

Despite these advantages, the remaining technical difficulty in robot-assisted mastectomy and reconstruction is to secure sufficient working space for robotic surgery. To overcome this difficulty, we used a modified Chung retractor. Selber et al. [12] described the use of carbon dioxide insufflation of the skin flap with $10 \mathrm{mmHg}$ of pressure, whereas Chung et al. [13] reported a robotic latissimus dorsi muscle harvest technique with a lighted retractor. Retraction provided more space for the robot arms than carbon dioxide insufflation; however, skin flap retraction may apply too much tension on the overlying mastectomy skin flap, which can result in skin flap ischemia and necrosis. Nonetheless, subcutaneous emphysema and hypercarbia can occur with carbon dioxide insufflation [14]. Further practical experience is needed to clarify which approach is more suitable for robotic mastectomy and reconstruction. In addition to securing the operative field with proper retraction, careful dissection and elevation of the medial side of the pectoralis muscle from the chest wall is required to prevent bleeding in perforators from the internal mammary artery. Once a perforator starts bleeding, the bleeding is harder to control than when the direct approach is used. Furthermore, due to chest wall curvature, the dissection of the sternal origin of the pectoralis major muscle is likely to be insufficient, which can cause lateral displacement of the implant.

The primary problem of robotic mastectomy and reconstruction is the high cost. The usual cost of a robotic system is more 
than US $\$ 2,000,000$, and that of the disposable instruments used in a single case is more than US $\$ 2,000$. Due to the high costs, further analysis of cost-effectiveness is needed [15].

In this article, we described our experiences and results. Further analyses of others' results are necessary to obtain reliable clinical evidence.

\section{NOTES}

\section{Conflict of interest}

No potential conflict of interest relevant to this article was reported.

\section{Ethical approval}

The study was approved by the Institutional Review Board of Severance Hospital (IRB No. 4-2018-0482) and performed in accordance with the principles of the Declaration of Helsinki. Institutional Review Board of our hospital waived informed consent and approved the design of this retrospective study.

\section{Patient consent}

The patients provided written informed consent for the publication and the use of their images.

\section{ORCID}

Sung Jae Ahn https://orcid.org/0000-0002-0324-0451 Seung Yong Song https://orcid.org/0000-0002-3145-7463 Hyung Seok Park https://orcid.org/0000-0001-5322-6036 Se Ho Park https://orcid.org/0000-0001-8089-2755 Dae Hyun Lew https://orcid.org/0000-0002-2625-5664 Tai Suk Roh https://orcid.org/0000-0001-8681-159X Dong Won Lee https://orcid.org/0000-0003-0046-3139

\section{REFERENCES}

1. Gerber B, Krause A, Dieterich M, et al. The oncological safety of skin sparing mastectomy with conservation of the nipple-areola complex and autologous reconstruction: an extended follow-up study. Ann Surg 2009;249:461-8.

2. Petit JY, Veronesi U, Rey P, et al. Nipple-sparing mastectomy: risk of nipple-areolar recurrences in a series of 579 cases. Breast Cancer Res Treat 2009;114:97-101.

3. Sakamoto N, Fukuma E, Higa K, et al. Early results of an endoscopic nipple-sparing mastectomy for breast cancer. Ann
Surg Oncol 2009;16:3406-13.

4. Leff DR, Vashisht R, Yongue G, et al. Endoscopic breast surgery: where are we now and what might the future hold for video-assisted breast surgery? Breast Cancer Res Treat 2011; 125:607-25.

5. Dziegielewski PT, Kang SY, Ozer E. Transoral robotic surgery (TORS) for laryngeal and hypopharyngeal cancers. J Surg Oncol 2015;112:702-6.

6. Toesca A, Peradze N, Manconi A, et al. Robotic nipple-sparing mastectomy for the treatment of breast cancer: feasibility and safety study. Breast 2017;31:51-6.

7. Toesca A, Peradze N, Galimberti V, et al. Robotic nipplesparing mastectomy and immediate breast reconstruction with implant: first report of surgical technique. Ann Surg 2017;266:e28-30.

8. Park HS, Lee DW, Kim JH, et al. The first case report of robot-assisted nipple sparing mastectomy and immediate reconstruction in Korea. In: Proceeding of Annual Meeting of Global Breast Cancer Conference 2017 (GBCC 2017); 2017 Apr 20-22; Jeju, Korea.

9. Kang SW, Chung WY. Transaxillary single-incision robotic neck dissection for metastatic thyroid cancer. Gland Surg 2015;4:388-96.

10. Kang SW, Jeong JJ, Yun JS, et al. Robot-assisted endoscopic surgery for thyroid cancer: experience with the first $100 \mathrm{pa}-$ tients. Surg Endosc 2009;23:2399-406.

11. Song HG, Yun IS, Lee WJ, et al. Robot-assisted free flap in head and neck reconstruction. Arch Plast Surg 2013;40: 353-8.

12. Selber JC, Baumann DP, Holsinger FC. Robotic latissimus dorsi muscle harvest: a case series. Plast Reconstr Surg 2012; 129:1305-12.

13. Chung JH, You HJ, Kim HS, et al. A novel technique for robot assisted latissimus dorsi flap harvest. J Plast Reconstr Aesthet Surg 2015;68:966-72.

14. Gottlieb A, Sprung J, Zheng XM, et al. Massive subcutaneous emphysema and severe hypercarbia in a patient during endoscopic transcervical parathyroidectomy using carbon dioxide insufflation. Anesth Analg 1997;84:1154-6.

15. Baik SH, Kwon HY, Kim JS, et al. Robotic versus laparoscopic low anterior resection of rectal cancer: short-term outcome of a prospective comparative study. Ann Surg Oncol 2009; 16:1480-7. 